\title{
Design e agroecologia impulsionando o empreendedorismo de base comunitária: caso Hackathon PBH - Gastronomia.
}

\author{
Bruna Almeida de Souza Andrade'; \\ Daniele Aparecida Santos Cipriano²; \\ Karen Magalhães Oliveira3; \\ Katia Andréa Carvalhaes Pêgo ${ }^{4}$;
}

resumo:

Este artigo tem como objetivo demonstrar como a associação entre o design, por uma perspectiva sistêmica, e a agroecologia pode impulsionar o empreendedorismo de base comunitária, além de promover a segurança alimentar em comunidades em contexto de vulnerabilidade. Importante ressaltar que a situação da insegurança alimentar, que foi se agravando nos últimos anos, registrou um aumento vertiginoso a partir da pandemia do coronavírus. Nesta conjuntura, é possível observar a atuação de diversos movimentos sociais, assim como do poder público que, em sintonia com os Objetivos de Desenvolvimento Sustentável (ODS) da ONU, criaram estratégias para monitorá-los e alocar recursos para projetos voltados ao tema. Como exemplo, podemos citar o 'Hackathon: Soluções para a Economia Criativa', realizado pela Prefeitura de Belo Horizonte (PBH) em parceria com a Pontifícia Universidade Católica de Minas Gerais (PUC Minas), no qual o objetivo foi incentivar a criação de soluções inteligentes, inovadoras e tecnológicas, para recuperar o setor criativo de $\mathrm{BH}$, no âmbito dos desafios impostos pela pandemia. O presente estudo de caso, desenvolvido durante este evento, teve como ponto de partida as 51 hortas urbanas mapeadas pela PBH. Após o diagnóstico deste sistema, foram elencados os problemas e as oportunidades, buscando-se avaliar o papel da agroecologia na melhoria das condições socioeconômicas dos agricultores familiares urbanos e da população do entorno, por meio da valorização dos saberes partilhados, dos serviços ecossistêmicos e dos equipamentos públicos. Os principais objetivos do projeto foram o fortalecimento dos negócios locais, a criação de novos produtos saudáveis e nutritivos, de novos serviços para atender ao sistema, de trabalho de qualidade e renda no próprio território. Como resultado apresentamos uma malha colaborativa que conecta cada horta comunitária com os empreendedores do seu entorno (restaurantes, bares, quitandas) e consumidores finais. Isto se dá por meio do web aplicativo "Afeto à mesa", que conecta as hortas, aos estabelecimentos parceiros por georreferenciamento e possibilita novas conexões de maneira orgânica e independente. Como consideração destaca-se a escalabilidade da solução proposta, pois o sistema de malha colaborativa inicialmente aplicado em um território piloto, pode ser replicado nas demais hortas urbanas, considerando o contexto de cada uma.

Palavras-chave: Malha colaborativa; perspectiva sistêmica; agroecologia; gastronomia; relações.

\footnotetext{
${ }^{1}$ Link do lattes: http://lattes.cnpq.br/0542137558402404

${ }^{2}$ Link do lattes: http://lattes.cnpq.br/1537336971041190

${ }^{3}$ Link do lattes: http://lattes.cnpq.br/5355142599846088

${ }^{4}$ Link do lattes: http://lattes.cnpq.br/1400735231277107
} 


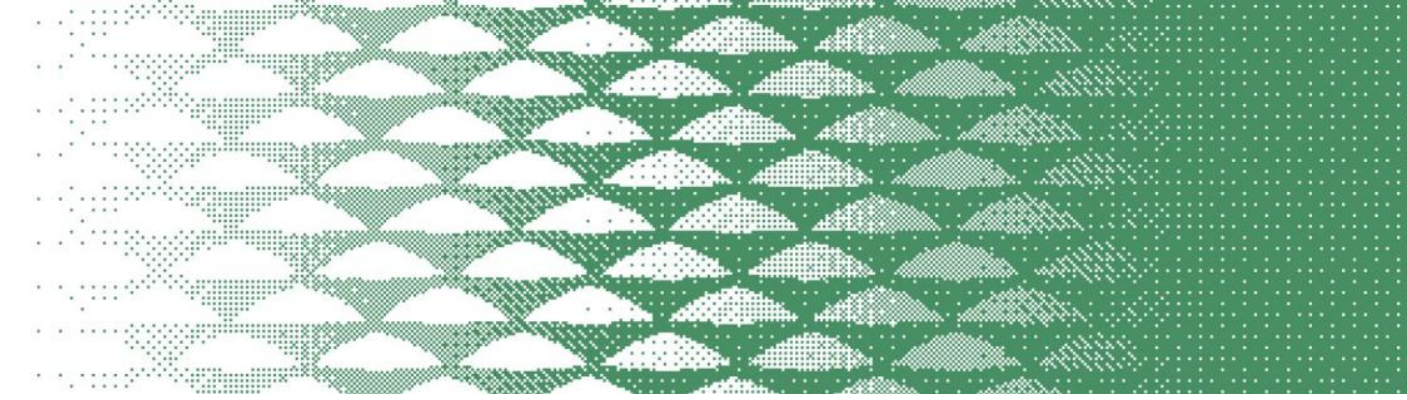

Espaço reservado para organização do congresso.

\section{Introdução}

O presente artigo apresenta um estudo de caso, cujo principais objetivos são: (i) demonstrar como a associação entre a agroecologia e as práticas colaborativas do design, por uma perspectiva sistêmica, pode impulsionar o empreendedorismo de base comunitária, em consonância com a promoção da segurança alimentar em comunidades vulneráveis da Região Metropolitana de Belo Horizonte (RMBH); (ii) descrever o processo de desenvolvimento do projeto realizado por uma equipe, de maneira colaborativa, durante o 'Hackathon: Soluções para a Economia Criativa' (de 17 a 24 de maio de 2021).

Este evento, promovido pela Prefeitura de Belo Horizonte (PBH) em parceria com a Pontifícia Universidade Católica de Minas Gerais (PUC Minas), teve como objetivo incentivar a criação de soluções inteligentes, inovadoras e tecnológicas, para recuperar o setor criativo de Belo Horizonte (BH), no contexto dos desafios impostos pela pandemia.

Em 2020, com a pandemia do Covid-19, foi possível observar que os índices de insegurança alimentar no Brasil se agravaram significativamente, se comparados aos anos anteriores. Como exemplo, podemos citar a redução de $85 \%$ do consumo de alimentos saudáveis entre os brasileiros. O relatório anual do Banco Mundial sobre pobreza e prosperidade compartilhada, indica que a pandemia deve acelerar ainda mais a volta do Brasil ao mapa da fome, pois muitas das famílias que já estavam em situação de insegurança alimentar encontraram maiores dificuldades em acessar uma alimentação de qualidade. Segundo pesquisa realizada pelo Food for Justice \#4 (2021), entre os alimentos não saudáveis consumidos durante a pandemia, o grupo dos doces registrou um aumento de $9 \%$. Por outro lado, a mesma pesquisa apontou uma redução de $44 \%$ no consumo de carnes, $36,8 \%$ no de hortaliças e legumes, $40,8 \%$ no de frutas e $40,4 \%$ no de queijos.

Nesse contexto, o projeto teve como pontos de partida as 51 hortas urbanas comunitárias implementadas pela PBH e a oportunidade de promover iniciativas que valorizassem o turismo de base comunitária em torno das hortas em questão. Considerou-se que, apesar das hortas comunitárias fazerem parte da Agenda de garantia da seguridade alimentar implementada pela PBH, muitas vezes, os moradores do território não se apropriam delas, provocando sua descontinuação. Sendo assim, foi proposta como solução, em um primeiro momento, uma malha colaborativa que conecta: (i) uma determinada horta comunitária, (ii) os empreendedores do seu entorno (restaurantes, bares, quitandas etc.) e (iii) os consumidores finais. A partir da implementação deste projeto piloto pretende-se escalar para toda a cidade, respeitando os limites de cada contexto, conectando todas as hortas por meio do aplicativo de celular proposto: "Afeto à mesa".

\section{Fundamentação teórica: agroecologia e segurança alimentar}

Desde a década de 1990, BH tem implementado diversas políticas públicas com o intuito de garantir segurança alimentar para todos. Em 2016 o orçamento investido chegou a 60,06 milhões e em 2020 a 82,6 milhões (SÁ, 2021). O desenvolvimento da agroecologia em Belo Horizonte deve-se, entre outros fatores, às redes da sociedade civil que contribuem com o poder público, por meio de legislações e equipamentos públicos em benefício da agricultura familiar urbana, sendo mais uma das iniciativas identificadas pela campanha 'Agroecologia nos Municípios', da Articulação Nacional de Agroecologia (ANA). Vários programas governamentais que começaram a se estruturar no município em torno da agroecologia como, por exemplo: Plantio em Espaços Alternativos, Hortas comunitárias, Hortas escolares, Centros de Vivência Agroecológica (CEVAEs), Programa Abastecer, Comboio do 
análise posterior, a equipe entendeu que os itens levantados apontam para problemas e oportunidades que se inter-relacionam.

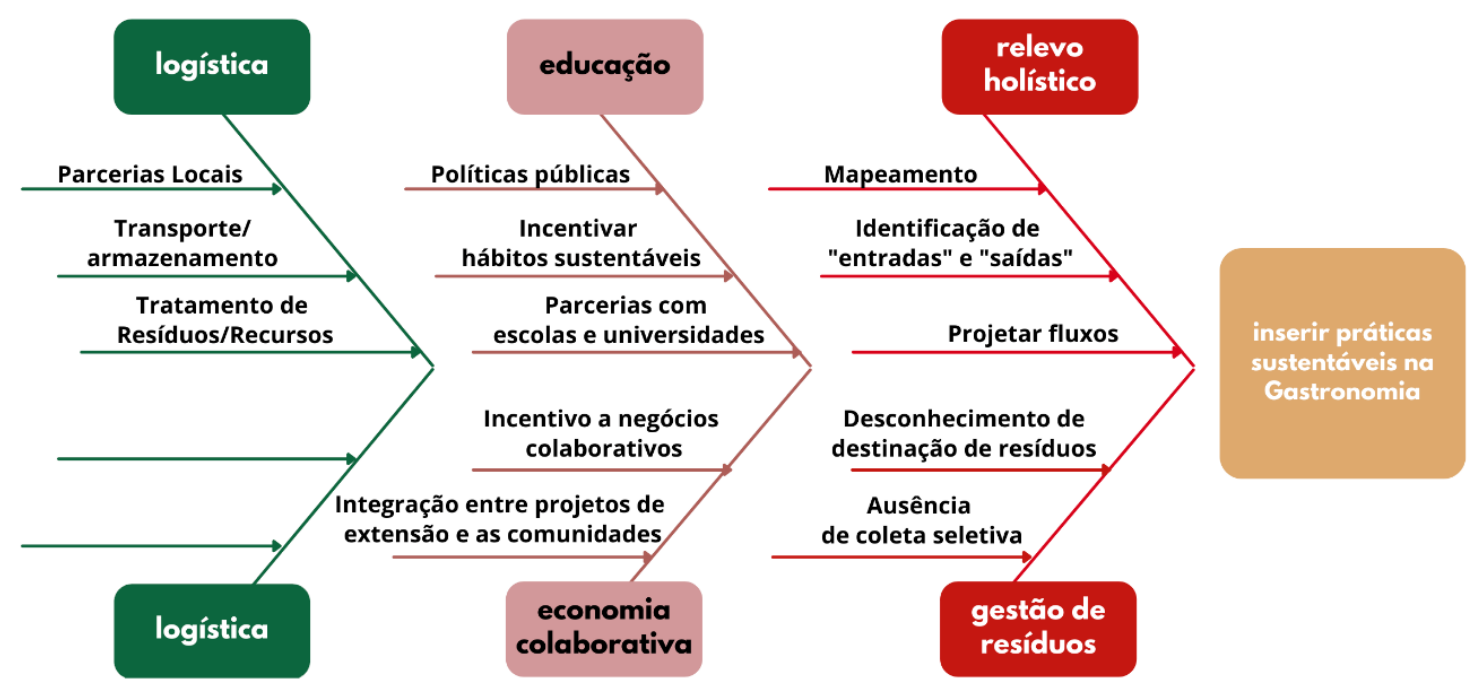

Gráfico 2: Espinha de Peixe.

Fonte: Elaborado pelas autoras

Como síntese desse processo, observou-se o fenômeno de "Não saber onde consumir", ou seja, não saber onde encontrar alimentação saudável com preços acessíveis. Dessa maneira, os sistemas agroecológicos coletivos destacam-se como um espaço em potencial para o fortalecimento das relações entre produtores, restaurantes (quitandas, bares etc.) e consumidores. Sendo assim, compreendeu-se que seria uma oportunidade em potencial o desenvolvimento de uma plataforma que estimulasse os fluxos de matéria e energia que alimentam esses sistemas, como demonstrado no Gráfico 3.

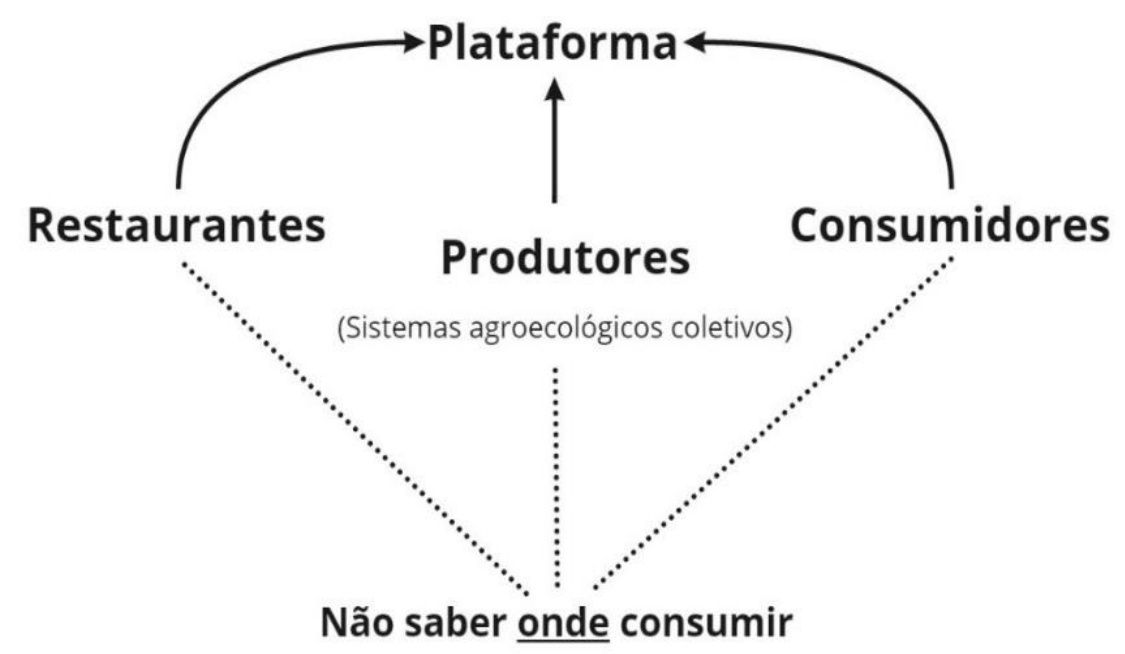

Gráfico 3: Síntese da solução proposta.

Fonte: Elaborado pelas autoras 


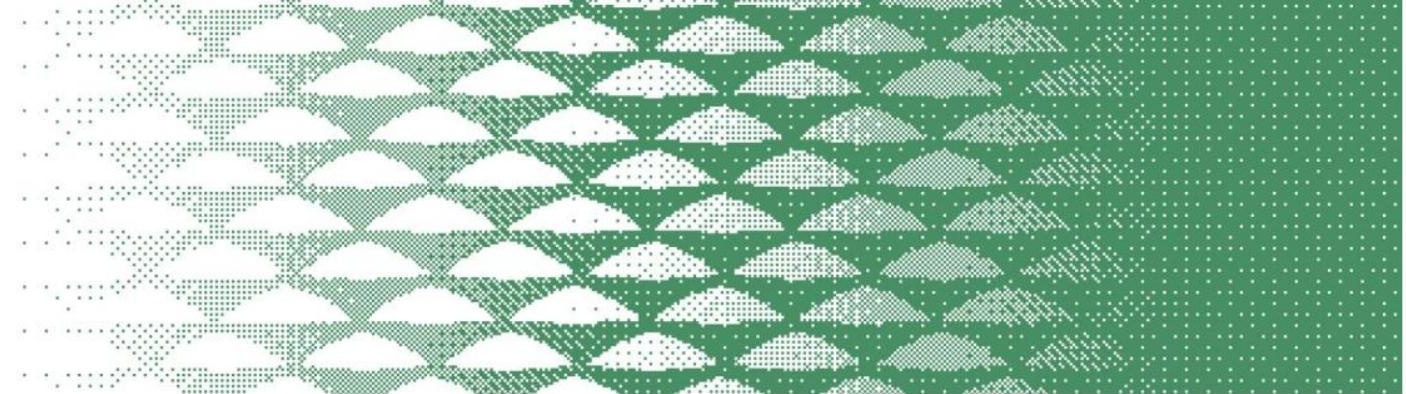

\subsection{Solução proposta}

Foi proposto então, uma malha colaborativa que pudesse conectar cada horta comunitária com os empreendedores do seu entorno (restaurantes, bares, quitandas) e consumidores finais, gerando o turismo de base comunitária e o fortalecimento da identidade do território como polo gastronômico. Como metodologia de implementação do projeto, foi proposto o desenvolvimento de um piloto (protótipo) em uma das 51 hortas comunitárias elencadas pela PBH, com o intuito de viabilizar a análise dos fluxos relacionais que a compõem, de acordo com a jornada do usuário, ilustrada no Gráfico 4.

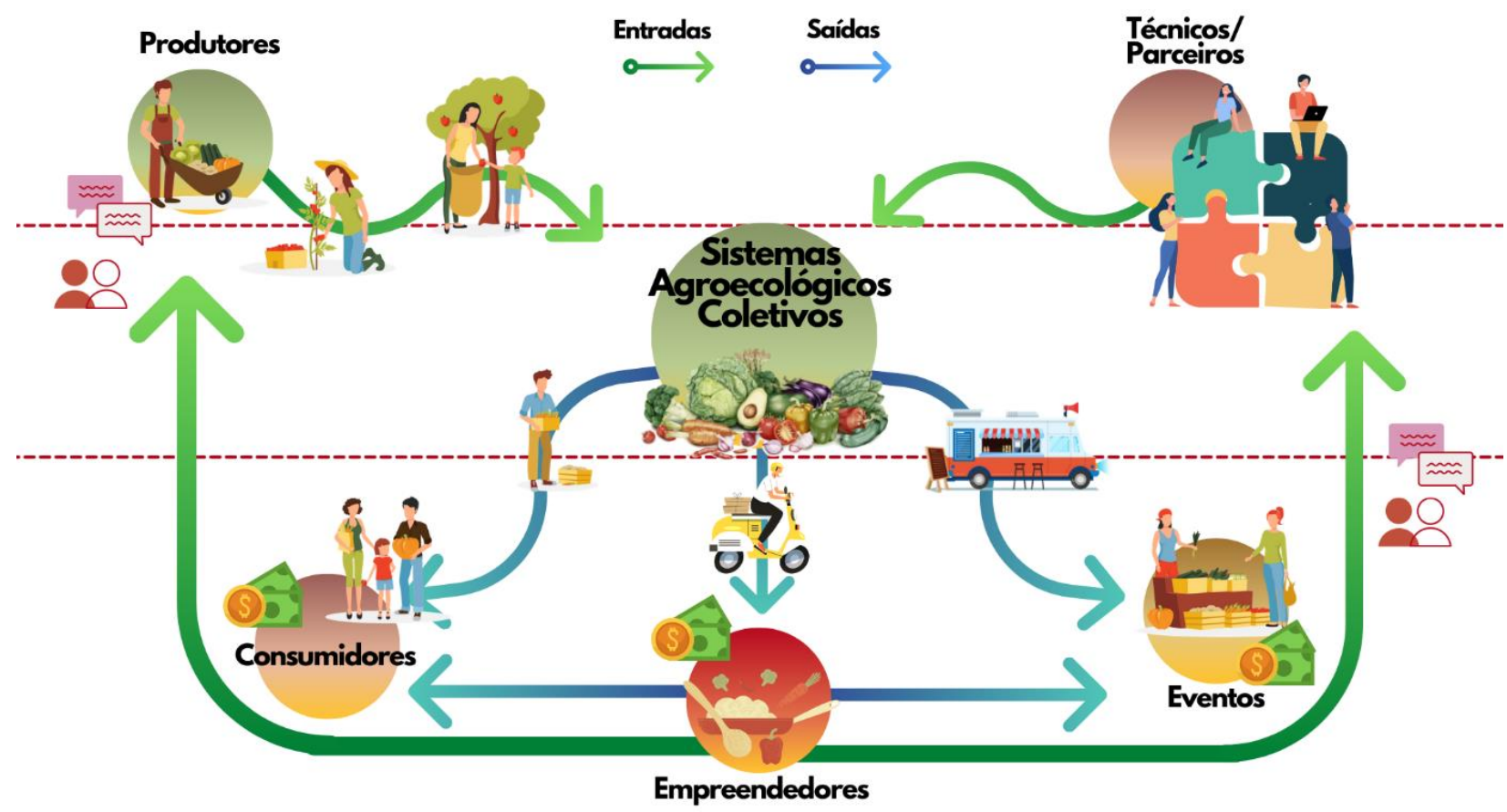

Gráfico 4: Jornada do usuário.

Fonte: Elaborado pelas autoras

Após a análise dos resultados do protótipo e suas possíveis atualizações, apresenta-se como proposta de desdobramento futuro possibilitar um maior alcance da população de $\mathrm{BH}$ aos benefícios socioambientais desse projeto, a partir do mapeamento dos fluxos de cada uma das 51 hortas comunitárias de BH e a posterior interconexão entre elas. Desta maneira, é possível escalar a oferta de alimentos saudáveis e nutritivos, assim como de seus serviços associados. Nesta perspectiva, vislumbrase a geração de novos postos de trabalho de qualidade e de geração de renda.

Como canal que promove tal conexão, o aplicativo denominado "Afeto à mesa" foi idealizado, considerando a interação por meio de um mapa, no qual consumidores podem localizar tanto as hortas comunitárias, quanto os empreendedores parceiros. Na Figura 1 são demonstradas algumas telas do protótipo, apresentado no último dia do evento. 

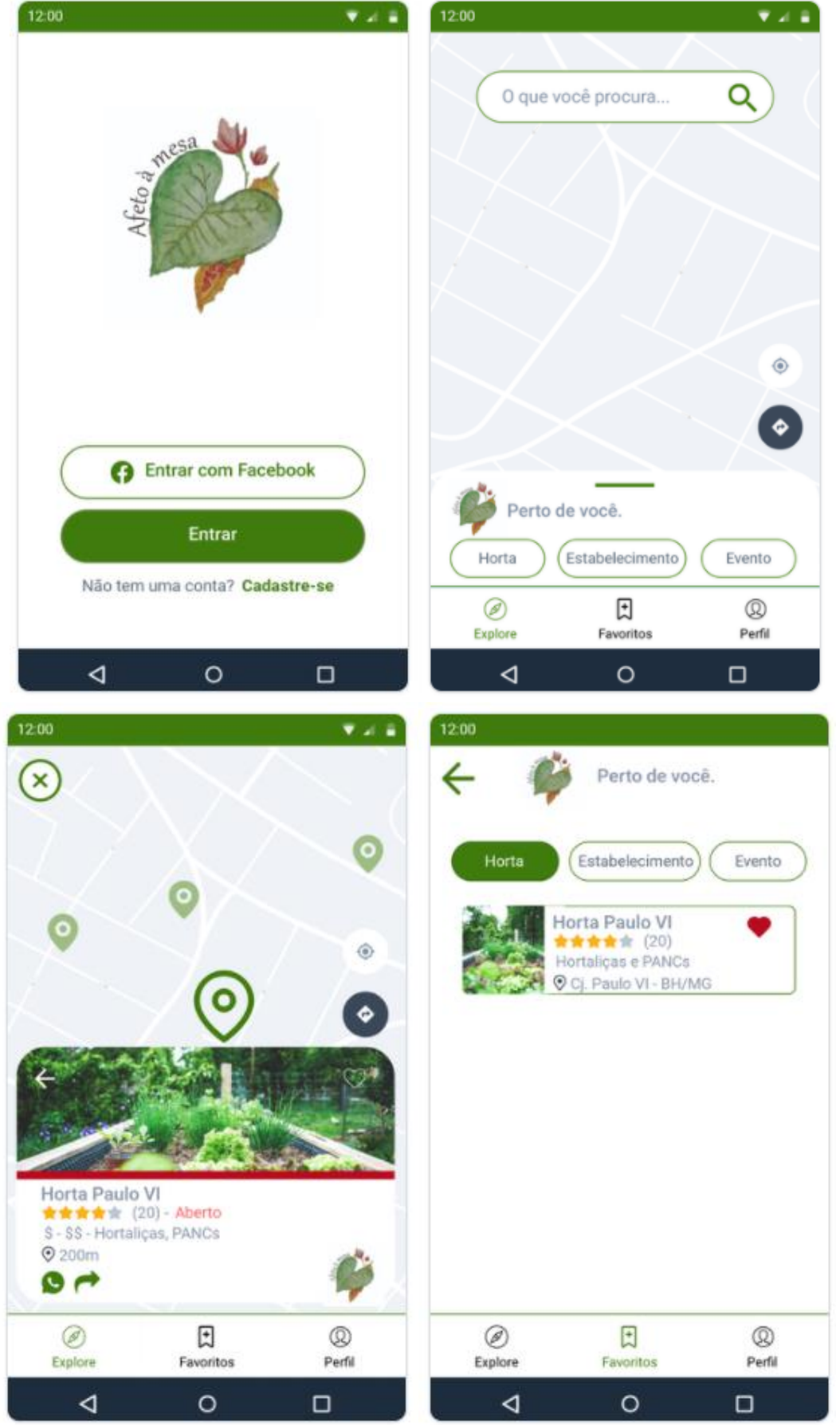

Figura 1: Telas do App 'Afeto a Mesa'.

Fonte: Elaborado pelas autoras

O principal elo da solução proposta é a parceria-chave entre os agricultores urbanos das hortas comunitárias já existentes com os potenciais novos parceiros, facilitando o escoamento do que é produzido e gerando fonte de receita aos hortelões, que apresentam dificuldades em dar continuidade aos trabalhos nas hortas por não saberem como e para quem vender suas produções. Além disso, o processo de construção das relações se dá de maneira contínua, assim como no conceito de 'malha' de 


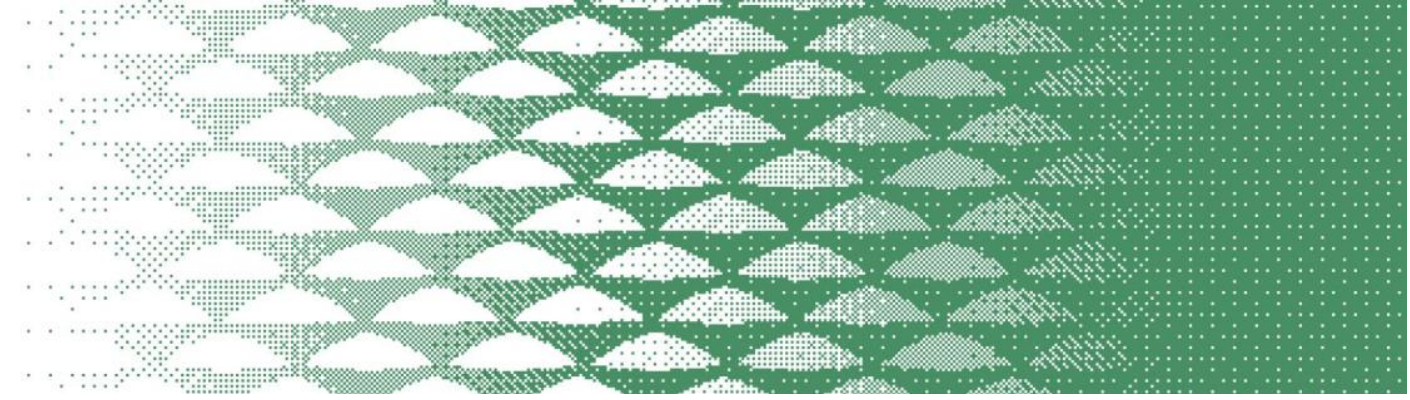

Tim Ingold (2012), em que os fluxos entre pessoas, sistemas e ambiente se dão em constante movimento de co-constituição, como coloca Munõz (2021).

A partir das conexões estabelecidas, diversas iniciativas de divulgação e fortalecimento da identidade cultural para o projeto foram propostas como, por exemplo: (i) comercialização de livros que reconheçam os saberes dos envolvidos; (ii) realização de circuitos abertos do tipo 'cozinha show', expandindo os horizontes de cozinheiros, chefs e produtores locais por toda $\mathrm{BH}$; (iii) produção e entrega de cestas semanais, contendo produtos tanto das hortas quanto dos cozinheiros parceiros; (iv) assim como diversas outras que possam ser planejadas e organizadas coletivamente. $\mathrm{O}$ roadmap, com as principais fases de implementação do projeto, se encontra representado no Gráfico 5.

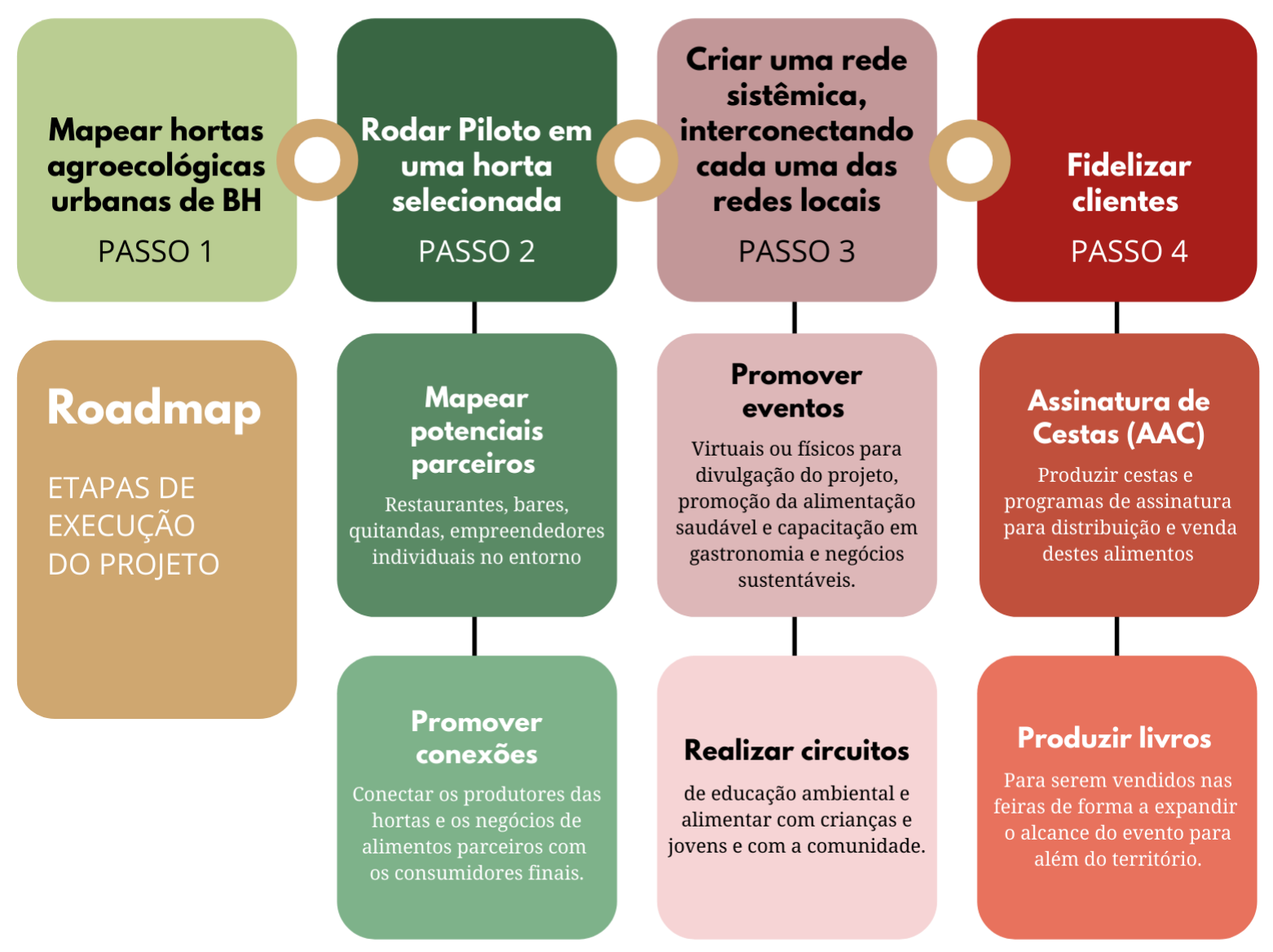

Gráfico 5: Roadmap - Próximos Passos.

Fonte: Elaborado pelas autoras

Os principais critérios para a seleção da solução proposta foram: (i) importância do tema Agricultura Urbana no contexto atual, pois discute a qualidade de vida nas cidades; (ii) segurança alimentar; (iii) oportunidade de fomentar a circulação nos sistemas agroecológicos de base coletivas/comunitárias já existentes em $\mathrm{BH}$; (iv) potencial de transformação social, que pode ser maximizado quando ocorre a apropriação pela população; (v) processo construtivo de pertencimento.

Nesta perspectiva, buscou-se também dar maior visibilidade às experiências gastronômicas na cidade, que foi eleita Cidade Criativa da Gastronomia pela Unesco, por meio da valorização os saberes culinários dos moradores de cada território, fortalecendo e potencializando as relações em comunidade e possibilitando o desenvolvimento de uma malha relacional que se sustente e amplie organicamente de maneira independente. 


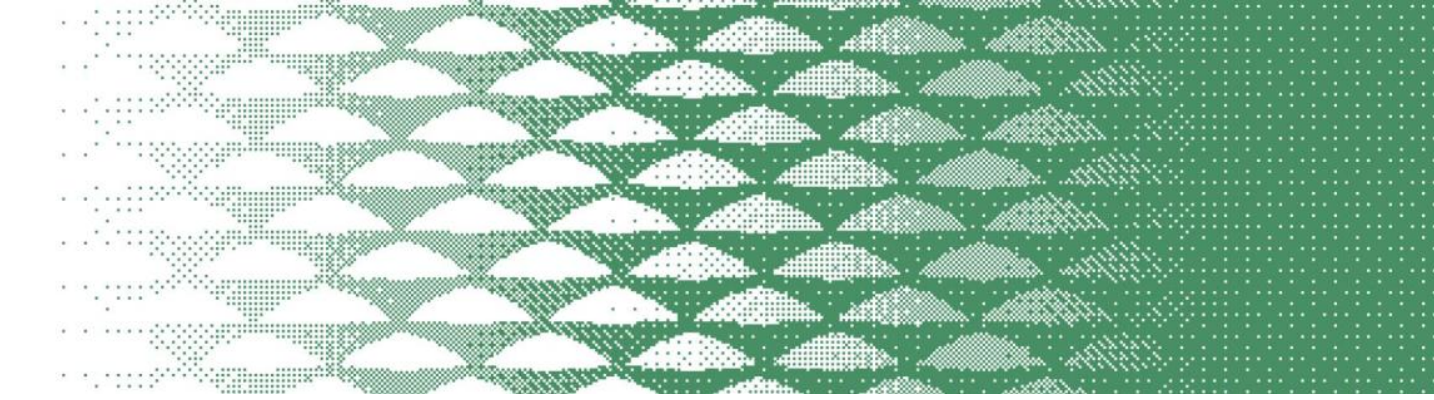

\section{Considerações finais}

Em linhas gerais percebeu-se como as metodologias de projeto colaborativas permitem ampliar as fronteiras do design, a partir da perspectiva sistêmica, que considera o território, os recursos e os saberes locais. Identificar as interações para além do conceito de 'rede', permitiu observar a multiplicidade de oportunidades e geração de valor, pouco explorados pelas comunidades em torno dos sistemas agroecológicos coletivos. Pode-se inferir, portanto, que a prática projetual que inclui ferramentas e métodos colaborativos é capaz de gerar novas oportunidades, de fortalecer o comércio de base comunitária e multiplicar a possibilidade de relações em territórios vulneráveis.

Contudo, vale destacar que esse é um processo contínuo de relacionamento com as comunidades e que pressupõe a práxis de transformação territorial, com base na consciência de classe e de lugar, o que demanda um tempo maior para a prática projetual gerar resultados duradouros (SAQUET, 2018). Contudo, pode indicar uma alternativa de desenvolvimento para sustentabilidade econômica, social, cultural e ambiental, pois atua com a lógica das pequenas iniciativas produtivas, preservando a cultura local e os recursos naturais. Trata-se de um processo, tal qual a 'malha' descrita por Ingold (2012), na qual cada linha se entrelaça, adicionando mais um fluxo de relações a cada contato, afetando e sendo afetado ininterruptamente, nesse complexo tecido da atualidade.

\section{Agradecimentos}

Agradecemos à Prefeitura de Belo Horizonte $(\mathrm{PBH})$, em especial à Secretaria Municipal de Desenvolvimento Econômico (SMDE), à PUC MINAS e ao seu Departamento de Relações Internacionais e à Organização das Nações Unidas para a Educação, a Ciência e a Cultura (UNESCO), pela oportunidade de participar do 'Hackathon - Soluções para a Economia Criativa'.

Agradecemos a habitual colaboração da equipe do Programa 'COMpasso: Parcerias Educacionais para Inovações em Comunidades', da Escola de Arquitetura da Universidade Federal de Minas Gerais (UFMG), que vem atuando sob a perspectiva das parcerias universidadesmunicipalidades-comunidades, com o intuito de atender às demandas destas últimas.

Agradecemos ainda à equipe do Projeto de Extensão 'Comunidades Criativas da RMBH na Perspectiva Sistêmica', coordenado pela professora e pesquisadora Kátia Andréa Carvalhaes Pêgo, da Escola de Design da Universidade do Estado de Minas Gerais (UEMG), que trabalhou intensamente para responder à altura dos desafios propostos.

\section{Design and agroecology driving community-based entrepreneurship: Hackaton PBH case - Gastronomy.}

\section{Abstract:}

This article aims to demonstrate how the association between design, from a systemic perspective, and agroecology can boost community-based entrepreneurship, in addition to promoting food security in communities in a vulnerable context. It is important to emphasize that the situation of food insecurity, which has been worsening in recent years, has registered a vertiginous increase from the coronavirus pandemic. At this juncture, it is possible to observe the performance of various social movements, as well as the government, which, in line with the UN Sustainable Development Goals (SDGs), created strategies to monitor them and allocate resources to projects focused on the theme. As an example, we can mention the 'Hackathon: Solutions for the Creative Economy', carried out by the Municipality of Belo Horizonte (PBH) in partnership with the Pontifical Catholic University of Minas Gerais (PUC Minas), in which the objective was to encourage the creation of solutions smart, innovative and technological, to recover the creative sector of $\mathrm{BH}$, within the scope of the challenges imposed by the pandemic. The present case study, developed during this event, had as its starting point the 51 urban gardens mapped 
by $\mathrm{PBH}$. After the diagnosis of this system, the problems and opportunities were listed, seeking to evaluate the role of agroecology in improving the socioeconomic conditions of urban family farmers and the surrounding population, through the valorization of shared knowledge, ecosystem services and services. public facilities. The main objectives of the project were the strengthening of local businesses, the creation of new healthy and nutritious products, new services to meet the system, quality work and income in the territory itself. As a result, we present a collaborative network that connects each community garden with the entrepreneurs in its surroundings (restaurants, bars, greengrocers) and end consumers. This happens through the web application "Affection at the table", which connects the gardens, to partner establishments by georeferencing and allows new connections in an organic and independent way. As a consideration, the scalability of the proposed solution is highlighted, as the collaborative mesh system initially applied in a pilot territory, can be replicated in other urban gardens, considering the context of each one.

Keywords: collaborative mesh; systemic perspective; agroecology; gastronomy; relations;

\section{Referências bibliográficas}

BISTAGNINO, L. Design sistemico: progettare la sostenibilità produttiva e ambientale. Torino: Slow Food Editore, 2009.

BLAY-PALMER, A.; SANTINI, G.; DUBBELING, M.; RENTING, H.; TAGUCHI, M.; GIORDANO, T. Validação da perspectiva de sistema alimentar cidade-região: promovendo sistemas alimentares cidade-região inclusivos e transformativos. In: PREISS, P. V.; SCHNEIDER, S. (Orgs.). Sistemas alimentares no século XXI DEBATES CONTEMPORÂNEOS. Porto Alegre: UFRGS, 2020. p. 51-88.

BROWN, T. D. T. Design thinking: uma metodologia poderosa para decretar o fim das velhas ideias. Rio de Janeiro: Campus, 2010.

GALINDO, E.; TEIXEIRA, M.A.; ARAÚJO M.; MOTTA, R.; PESSOA, M.; MENDES, L.; RENNÓ, L. Efeitos da pandemia na alimentação e na situação da segurança alimentar no Brasil. Berlin: Food for Justice: Power, Politics, and Food Inequalities in a Bioeconomy, 2021. n. 4. Disponível em: https://refubium.fu-berlin.de/handle/fub188/29813. Acesso em: 12 jun. 2021.

INGOLD, T. Trazendo as coisas de volta à vida: emaranhados criativos num mundo de materiais. Porto Alegre: Horizontes Antropológicos [online], 2012. n. 37, p. 25-44. Disponível em: https://doi.org/10.1590/S0104-71832012000100002. Acesso em: 29 maio 2021.

ISHIKAWA, K. Controle de qualidade total: à maneira japonesa. Rio de Janeiro: Campus, 1995.

LE COQ, J. F.; PATROUILLEAU, M.M.; SABOURIN, E.; NIEDERLE, P.A. A institucionalização das políticas públicas para a agroecologia e produção orgânica na América Latina. In: PREISS, P. V.; SCHNEIDER, S. (Orgs). Sistemas alimentares no século XXI DEBATES CONTEMPORÂNEOS. Porto Alegre: UFRGS, 2020. p. 257-266.

MUNOZ, S. Un diálogo entre la red de Bruno Latour y la malla de Tim Ingold cruzado por la experiencia. Santiago: Cinta moebio, 2021. n. 70, p. 68-80. Disponível em: https://scielo.conicyt.cl/scielo.php?pid=S0717-554X2021000100068\&script=sci_arttext. Acesso em: 01 jun. 2021.

SAQUET, M. A descoberta do território e outras premissas do desenvolvimento territorial. São Paulo: Revista Brasileira de Estudos Urbanos e Regionais, 2018. v. 20, n. 3, p. 479-505. Disponível em: https://www.scielo.br/pdf/rbeur/v20n3/2317-1529-rbeur-20-03-479.pdf. Acesso em: 28 maio 2021. 